\title{
The post-trial effect of oral periodic presumptive treatment for vaginal infections on the incidence of bacterial vaginosis and Lactobacillus colonization
}

\author{
Jennifer E. Balkus, MPH ${ }^{1}$, Walter Jaoko, $\mathrm{MBChB}$, MMed, $\mathrm{PhD}^{2}$, Kishorchandra Mandaliya, \\ $\mathrm{MBChB}^{3}$, Barbra A. Richardson, $\mathrm{PhD}^{4,5,7}$, Linnet Masese, MBChB, MPH ${ }^{1}$, Ruth Gitau, \\ MBChB $^{2}$, James Kiarie, MBChB, MMed, MPH${ }^{2}$, Jeanne Marrazzo, MD, MPH ${ }^{6}$, Carey \\ Farquhar, MD, $\mathbf{M P H}^{1,6,7}$, and R. Scott McClelland, MD, $\mathbf{M P H}^{1,2,6,7}$ \\ ${ }^{1}$ University of Washington, Department of Epidemiology;Seattle, WA \\ 2University of Nairobi, Nairobi, Kenya \\ ${ }^{3}$ PathCare, Mombasa, Kenya \\ ${ }^{4}$ University of Washington, Department of Biostatistics;Seattle, WA \\ ${ }^{5}$ Fred Hutchinson Cancer Research Center, Vaccine and Infectious Disease Division; Seattle, WA \\ ${ }^{6}$ University of Washington, Department of Medicine;Seattle, WA \\ ${ }^{7}$ University of Washington, Department of Global Health; Seattle, WA
}

\begin{abstract}
Background-We previously demonstrated a decrease in bacterial vaginosis (BV) and an increase in Lactobacillus colonization among randomized controlled trial (RCT) participants who received monthly oral periodic presumptive treatment (PPT) $[2 \mathrm{~g}$ metronidazole $+150 \mathrm{mg}$ fluconazole]. Post-trial data were analyzed to test the hypothesis that the treatment effect would persist following completion of one year of PPT.
\end{abstract}

Methods-Data were obtained from women who completed all 12 RCT visits and attended $\geq 1$ post-trial visit within 120 days following completion of the RCT. We used Andersen-Gill proportional hazards models to estimate the post-trial effect of the intervention on the incidence of BV by Gram stain and detection of Lactobacillus species by culture.

Results-The analysis included 165 subjects ( 83 active and 82 placebo). The post-trial incidence of BV was 260 per 100 person-years in the intervention arm versus 358 per 100 person-years in the placebo arm (hazard ratio $[\mathrm{HR}]=0.76 ; 95 \%$ confidence interval $[\mathrm{CI}]: 0.51-1.12$ ). The post-trial incidence of Lactobacillus colonization was 180 per 100 person-years in the intervention arm versus 127 per 100 person-years in the placebo arm (HR=1.42; 95\% CI: 0.85-2.71).

Conclusions-Despite a decrease in BV and an increase in Lactobacillus colonization during the RCT, the effect of PPT was not sustained at the same level following cessation of the

Correspondence: Jennifer E. Balkus, University of Washington, Box 359909, 325 9th Avenue, Seattle, WA 98104, jbalkus@u.washington.edu, Telephone: 206-685-8219, Fax: 206-543-4818.

Please address reprint requests to above author.

This abstract was presented at the International Society for Sexually Transmitted Disease Research Conference in Quebec City, Canada; July 10-13, 2011.

RS McClelland has received honoraria and a donation of study product for an ongoing clinical trial from Embil Pharmaceutical Company. JM Marrazzo receives research funding from Cepheid. All other authors declare no commercial or other associations that might pose a conflict of interest. 
intervention. New interventions that reduce BV recurrence and promote Lactobacillus colonization without the need for ongoing treatment are needed.

\section{Keywords}

Bacterial vaginosis; Lactobacillus; periodic presumptive treatment; suppressive treatment

\section{Introduction}

Bacterial vaginosis (BV) is a common vaginal infection that is associated with an increased risk of HIV-1 acquisition, other sexually transmitted infections (STIs), and adverse reproductive health outcomes. ${ }^{1-5}$ Though there are several bacterial species that are frequently detected among women with $\mathrm{BV}$, the exact etiology of this condition remains unknown. Standard antibiotic regimens for treatment of BV result in cure rates $>75 \%$. 6,7 However, approximately one-third of women will experience another episode of BV within 3 months of treatment and more than half will have a recurrence within one year. ${ }^{8-10}$ Given the high prevalence of BV and the frequency with which it recurs, alternative treatment regimens are needed that reduce the incidence and recurrence of BV.

Suppressive antibiotic therapy has been shown to effectively reduce BV recurrence. A clinical trial compared topical twice-weekly administration of $0.75 \%$ metronidazole vaginal gel versus placebo among women who were successfully treated for BV at enrollment. ${ }^{9}$ This trial demonstrated significant reductions in BV among women in the intervention arm after 16 weeks of study product use. Following the completion of suppressive therapy, the probability of recurrent BV by Amsel's criteria ${ }^{11}$ was $30 \%$ in the intervention arm versus $61 \%$ in the placebo arm ( $\mathrm{p}=0.001)$. Unfortunately, the effect of the intervention did not persist in the absence of treatment. After 12 weeks of post-intervention follow-up, the probability of recurrent BV was $66 \%$ in the intervention arm versus $82 \%$ in the placebo arm.

It is possible that longer durations of suppressive therapy are required to facilitate changes in the vaginal microbiota that will persist following the completion of treatment, resulting in lower rates of BV recurrence. Previously, we conducted a randomized controlled trial (RCT) of suppressive therapy using oral periodic presumptive treatment (PPT) to reduce vaginal infections among Kenyan women. ${ }^{12}$ We observed a decrease in BV (hazard ratio [HR] = 0.55 ; 95\% confidence interval [CI], 0.49-0.63) and an increase in Lactobacillus colonization $(\mathrm{HR}=1.47 ; 95 \% \mathrm{CI}, 1.19-1.80)$ among women randomized to receive oral $2 \mathrm{~g}$ metronidazole plus $150 \mathrm{mg}$ fluconazole monthly for 12 months. After the trial, women were invited to continue follow-up in an open cohort study. Given the significant reduction in BV and increase in Lactobacillus colonization achieved after 12 months of PPT, we wondered if this intervention might have created vaginal conditions that would result in continued normal vaginal microbiota after the intervention was completed. Therefore, we analyzed post-trial data to test the hypothesis that the treatment effect would persist in the absence of PPT.

\section{Materials and Methods}

Female sex workers enrolled in a prospective, open cohort study of risk factors for HIV-1 acquisition were recruited to participate in the RCT. ${ }^{13}$ Detailed methods for the RCT have been published. ${ }^{12}$ Briefly, the trial was conducted in Mombasa, Kenya between May 2003 and December 2006 and enrolled women if they were 18-45 years of age, not pregnant, HIV-1-seronegative and had no abnormal vaginal discharge or itching at enrollment. Both the open cohort study and the RCT received approval from the institutional review boards at Kenyatta National Hospital/University of Nairobi (Nairobi, Kenya) and the University of 
Washington (Seattle, USA). All participants provided separate written informed consent for the cohort study and the RCT.

At enrollment in the RCT and each of 12 monthly follow-up visits, a face-to-face interview was conducted to collect information on medical history, sexual history and vaginal washing practices. A physical examination, including speculum-assisted pelvic examination, was performed with collection of specimens for diagnosis of genital tract infections. A urine pregnancy test was performed and blood was collected for HIV-1 testing. Participants were randomized to receive oral $2 \mathrm{~g}$ of metronidazole plus $150 \mathrm{mg}$ of fluconazole or identical placebo. At monthly visits, study product was administered orally as directly observed treatment. Women reporting abnormal vaginal discharge or vulvovaginal itching were treated syndromically with a single dose of $2 \mathrm{~g}$ oral metronidazole plus clotrimazole $200 \mathrm{mg}$ vaginal suppositories nightly for 3 nights. Study product was withheld when this treatment was dispensed. Other genital tract infections were treated according to World Health Organization and Kenya Ministry of Health guidelines. At completion of the RCT, women were invited to resume follow-up in the open cohort study. Women who resumed follow-up continued with standard procedures for the cohort, which were similar to those conducted during the RCT.

All laboratory procedures for the RCT and the cohort study were performed in Mombasa, Kenya. A Gram stain of vaginal fluid was evaluated for diagnosis of BV by Nugent criteria. ${ }^{14} \mathrm{~A}$ vaginal saline wet mount was examined microscopically for the presence of motile trichomonads and fungal elements. A drop of $10 \%$ potassium hydroxide was added to the slide and evaluated again for the presence of yeast buds or hyphae. Trichomonas vaginalis culture was performed in Diamond's modified medium. Lactobacillus culture was performed on Rogosa agar. ${ }^{15}$ Subculture of Lactobacillus isolates on tetramethylbenzdine agar containing horseradish peroxidase was performed to evaluate hydrogen peroxide $\left(\mathrm{H}_{2} \mathrm{O}_{2}\right)$ production. ${ }^{16}$ Endocervical secretions were cultured on modified Thayer-Martin media for $N$. gonorrhoeae. A Gram stain of endocervial secretions was evaluated for cervicitis, defined as the presence of an average of $\geq 30$ polymorphonuclear leukocytes per high-power field on microscopic examination (original magnification X100). HIV-1 testing was performed using an ELISA (Detect-HIV [BioChem ImmunoSystems]). Positive ELISA results were confirmed using a second ELISA (Recombigen [Cambridge Biotech] or Vironostika [bioMerieux]).

The objective of this analysis was to test the hypothesis that the treatment effect would persist following completion of one year of PPT. The study population consisted of nonpregnant, HIV-1-seronegative women who completed all 12 RCT visits and attended $\geq 1$ cohort study visit within 120 days of their final RCT visit. We decided a priori to include only the first 3 visits that occurred within the 120-day post-trial period. We used descriptive statistics, chi-squared tests for categorical outcomes and Wilcoxon rank sum tests for continuous outcomes to summarize and compare demographic and clinical characteristics by study arm. The same methods were used to compare women included in the analysis versus those excluded (factors evaluated are listed in Table 1). Andersen-Gill proportional hazards models that allow for recurrent events were used to estimate the post-trial effect of the intervention versus placebo on our primary outcomes: the incidence of BV by Gram stain (Nugent score 27 ) and any Lactobacillus species by culture. Secondary outcomes included abnormal vaginal flora (Nugent score $>3$ ) and $\mathrm{H}_{2} \mathrm{O}_{2}$-producing Lactobacillus species by culture. Demographic and clinical characteristics that were unbalanced by study arm at the final RCT visit ( $\leq 0.10$ ), which served as the baseline visit for this analysis, were included in the final statistical models. To better understand the effect of the intervention on BV during and after the trial, we conducted a descriptive analysis in which we calculated the incidence of BV at each RCT and post-trial follow-up visit. All statistical tests were assessed 
using a 2-sided alpha of 0.05. Analyses were conducted using Stata version 11.0 (StataCorp, Inc., College Station, TX).

\section{Results}

The RCT enrolled 310 participants (155 per arm), of whom 208 completed all 12 RCT visits. Of these 208, 165 participants ( 83 intervention and 82 placebo) returned for a cohort study visit within 120 days of their last RCT visit and were included in this analysis. Participants who returned within 120 days were slightly older (median (IQR): 34 years (30 40 ) versus 31 year $(27-36) ; \mathrm{p}<0.001)$ and reported a longer duration of sex work (median (IQR): 7 years $(3-12)$ versus 4 years $(2-7) ; \mathrm{p}<0.001)$ compared to those who did not return following completion of the RCT.

All participants included in this analysis had at least one post-trial follow-up visit within 120 days of their last RCT visit, 147 participants had two visits (72 intervention versus 75 placebo) and 103 had three visits (56 intervention versus 47 placebo). The minimum time to the first post-trial visit was 25 days in the intervention arm and 26 days in the placebo arm. At the final RCT visit, demographic and behavioral characteristics were generally similar by study arm (Table 1). However, there was a trend towards a higher proportion of women in the intervention arm reporting vaginal washing in the last week compared to women in the placebo arm (94\% versus $85 \%$; $\mathrm{p}=0.10$ ). Clinical factors were similar by arm, with the exception of BV and abnormal vaginal flora. The prevalence of $\mathrm{BV}$ at the final RCT visit was $17 \%$ in the intervention arm and $43 \%$ in the placebo arm ( $p<0.001)$ and the prevalence of abnormal vaginal flora was $33 \%$ in the intervention arm and $61 \%$ in the placebo arm $(\mathrm{p}<0.001)$

The post-trial incidence of BV, abnormal vaginal flora, any Lactobacillus colonization and $\mathrm{H}_{2} \mathrm{O}_{2}+$ Lactobacillus colonization are presented in Table 2. In the post-trial period, we observed a lower incidence of BV (260 per 100 person-years versus 358 per 100 personyears) and a higher incidence of any Lactobacillus colonization (180 per 100 person-years versus 127 per 100 person-years) among women receiving the intervention compared to women receiving the placebo, respectively. However, the differences were not statistically significant (Table 2). The findings were similar for abnormal vaginal flora (420 per 100 person-years versus 536 per 100 person-years) and $\mathrm{H}_{2} \mathrm{O}_{2}+$ Lactobacillus colonization (77 per 100 person-years versus 61 per 100 person years). The results did not differ in multivariable models adjusting for vaginal washing in the past week. To better understand the effect of the intervention over time in the study population, we calculated the incidence of BV at each visit interval during the RCT and in the post-trial interval by study arm. Although we observed an overall decrease in the incidence of BV in the intervention arm during the trial, following the cessation of the intervention, the incidence of $\mathrm{BV}$ in the intervention arm increased during the post-trial period (Figure 1). Thus, the effect of the intervention appears to wane following cessation of the intervention.

\section{Discussion}

In this analysis of post-trial data, the effect of monthly PPT with $2 \mathrm{~g}$ metronidazole plus $150 \mathrm{mg}$ fluconazole was not sustained during the 120 days following cessation of the intervention. In the RCT, we observed a $45 \%$ reduction in the risk of BV and a $47 \%$ increase in the likelihood of Lactobacillus colonization in the intervention arm. ${ }^{12}$ Though the incidence of BV was lower in the intervention arm than the placebo during the post-trial period, the magnitude of the difference was less than what was observed during the RCT. In addition, we observed that the incidence of $\mathrm{BV}$ in the placebo arm decreased following the completion of the trial (Figure 1). This decrease might be due to chance and could reflect normal fluctuations in the vaginal microbiota. More importantly, we observed a gradual 
increase in the post-trial incidence in the intervention arm, which suggests that the suppressive effect of the intervention on BV diminished following cessation. Conversely, the magnitude of the difference in Lactobacillus colonization was similar to what was observed during the RCT. However, fewer participants and a shorter duration of follow-up contributed to a reduction in study power resulting in a difference in Lactobacillus colonization that was not statistically significant. Vaginal washing, which has been associated with an increased risk of $\mathrm{BV}^{17,18}$, differed slightly by arm in the post-trial period, though adjustment for this behavior produced similar results. Our findings are similar to those of a trial of suppressive therapy using biweekly $0.75 \%$ metronidazole vaginal gel. ${ }^{9}$ The reduced effect of PPT and suppressive therapy following the completion of treatment highlights the need for new interventions that reduce BV recurrence and promote Lactobacillus colonization without the need for ongoing treatment.

Advances in our ability to characterize the vaginal microbiota using cultivation-independent methods, such as fluorescent in situ hybridization (FISH) and 16S rRNA polymerase chain reaction (PCR) assays, have shed light on microbiological factors associated with BV recurrence and persistence. ${ }^{19-22}$ Recent evidence suggests that the high rate of recurrent BV could be due to the formation of adherent biofilms of BV-associated bacteria. ${ }^{19,20} \mathrm{An}$ evaluation of vaginal biopsies from women with and without BV by Amsel's criteria showed that a dense bacterial biofilm was observed using FISH among $90 \%$ of biopsy specimens from subjects with BV and $10 \%$ of subjects without BV $(\mathrm{p}<0.001) .{ }^{19}$ These biofilms were composed primarily Gardnerella and Atobobium, two species highly associated with BV, ${ }^{22-24}$ A follow-up study among women with BV (positive by both Amsel's criteria and Nugent's score) who were treated with 500mg metronidazole twice daily for 7 days reported a decrease in biofilms in the 7 days following treatment. However, despite decreases in Nugent score $(<7)$ and the absence of symptoms among all participants, the biofilm re-emerged in the 5 weeks following treatment. ${ }^{20}$ In addition, cultivationresistant species identified through species-specific 16S rRNA PCR assays have been associated with BV persistence. ${ }^{21}$ Among women with symptomatic BV who were treated with vaginal metronidazole gel, women with persistent $\mathrm{BV}$ one month post-treatment were more likely to have BVAB1, BVAB2, BVAB3, Megasphaera, and Peptoniphulus lacrimalis detected in vaginal samples at baseline compared to women who experienced clinical cure (defined as $<3$ Amsel's criteria).

Eradication or more profound reduction in the concentrations of certain critical BV-associate species might be required to significantly reduce rates of $\mathrm{BV}$ recurrence after treatment. This conceivably could be accomplished by higher doses and/or longer courses of antibiotic treatment. Low cure and high recurrence rates of BV were observed with single dose oral $2 \mathrm{~g}$ metronidazole, leading to a change in treatment guidelines to multi-day regimens that deliver a higher total dose over a longer duration. ${ }^{6}$ However, the cure and recurrence rates with the currently recommended regimens remain unacceptably low and high, respectively. As a result, alternative antibiotic regimens continue to be evaluated. A trial of topical therapy demonstrated that metronidazole $500 \mathrm{mg}$ plus nystatin 100,000 units (co-formulated suppositories) administered for 5 nights was superior to metronidazole $0.75 \%$ gel (containing $37.5 \mathrm{mg}$ of metronidazole per dose) also administered for 5 nights. ${ }^{25}$ In this study, BV (positive by both Amsel's criteria and Nugent's score) was detected at the first follow-up visit among $7 \%$ of women in the metronidazole plus nystatin arm compared to $26 \%$ in the metronidazole gel arm (relative risk $=0.28$ [95\% CI: $0.11-0.71]$ ). The authors also assessed BV-free survival following treatment. At 104 days following treatment, 33\% of women in the metronidazole plus nystatin arm had experienced a recurrent episode of BV compared to $52 \%$ of women in the metronidazole arm $(\mathrm{p}=0.01)$. Though the rate of BV recurrence was lower in the intervention arm, it is similar to what has been reported by others at approximately 3 months post-treatment with oral metronidazole. ${ }^{8}$ 
Non-antibiotic approaches to improve BV cure rates and reduce recurrence have also been investigated. With the depletion of lactobacilli commonly observed among women with BV, there is great interest in the use of probiotic regimens to facilitate re-colonization of the vagina with Lactobacillus species. ${ }^{26}$ It is hypothesized that Lactobacillus colonization, in particular colonization with $\mathrm{H}_{2} \mathrm{O}_{2}$-producing strains, may be responsible for maintaining a low vaginal $\mathrm{pH}$ and controlling the growth of other bacteria through their ability to produce lactic acid and $\mathrm{H}_{2} \mathrm{O}_{2} \cdot{ }^{16,27}$ A number of small studies have evaluated probiotic regimens administered either alone or in combination with antibiotics or estriol for $\mathrm{BV}$ treatment ${ }^{28}$; however, additional evidence is needed to support the use of probiotics for BV treatment. Continued development and rigorous evaluation of treatment regimens, both antibiotic and probiotic, that further improve the cure rate and reduce the recurrence rate of $\mathrm{BV}$ are necessary.

The findings from this analysis should be interpreted in the context of several limitations. This is a secondary analysis in which we selected a sub-group of participants from the RCT. Our selection criteria for this analysis resulted in the exclusion of a large number of women either due to incomplete RCT follow-up ( $<12$ visits) or no follow-up visit within the posttrial period. We chose to restrict our analysis to women who completed all 12 follow-up visits, since we hypothesized that if there was a true post-trial effect of the intervention we would be most likely to observe it in this population. By design, our selection criteria resulted in a smaller sample size. In addition, this analysis included a relatively short duration of post-trial follow-up. As a result, we cannot rule out the persistence of small differences in the incidence of BV and Lactobacillus colonization by study arm in the posttrial period. There is the potential for selection bias in sub-group analyses. However, we are reassured by the fact that women who were included in the analysis were generally similar to those excluded. Moreover, among women included in this sub-group analysis, there were minimal differences by study arm. Behavioral characteristics that are unique to the study population could also limit the generalizability of our findings. Women in our study reported high rates of vaginal washing. The post-trial effect of the intervention may differ in populations who report less vaginal washing. Our characterization of the vaginal microbiota was limited to detection of Lactobacillus by culture. Therefore, we were unable to assess the effect of the intervention in the post-trial period on other vaginal bacterial species, including $\mathrm{BV}$-associated bacteria. Lastly, we used a single dose metronidazole regimen, which was the recommended treatment during the time that the study was conducted. Currently, single dose regimens are no longer recommended and have been replaced by multi-day regimens as the preferred treatment for BV. ${ }^{6}$

Our findings raise several important points for consideration in future research studies. First, future studies of periodic presumptive or suppressive treatment for BV should continue to assess the effect of intervention following cessation of the treatment regimen. It is unlikely that interventions that require continued treatment will be feasible or acceptable to patients. Therefore, it is critical to evaluate the sustained effect of the intervention following an extended course of suppressive therapy. Second, our study employed culture methods alone to characterize the vaginal microbiota since cultivation-independent methods (16S rRNA PCR) were not widely available at the time the RCT was conducted. With the increasing availability of cultivation-independent methods, future studies may be strengthened through the use of both culture and cultivation-independent methods. Due to the polymicrobial nature of $\mathrm{BV}$ and the importance of cultivation-resistant species in this condition, it is critical to understand the immediate and long-term effect of treatment on both cultivatable and cultivation-resistant vaginal bacterial species. Culture and cultivation-independent methods each have strengths and weakness. ${ }^{29}$ The use of combined methods allows for a more complete characterization of the vaginal microbiota and may provide new insights into mechanisms of BV cure, persistence and recurrence. 
In summary, PPT is a promising approach for reducing the incidence of BV and increasing Lactobacillus colonization. However, the regimens evaluated to date have demonstrated a reduced effect following the cessation of therapy. New interventions that decrease BV recurrence and promote long-term Lactobacillus colonization without the need for ongoing PPT or suppressive therapy are needed.

\section{Acknowledgments}

This research was supported by a grant from the National Institutes of Health [grant K23 AI52480]. JE Balkus was supported by a grant from the University of Washington Center for AIDS and STDs [grant T32 AI007140-32]. C Farquhar is supported in part by a grant from the National Institutes of Health [grant K24 AI087399]. Additional support for the Mombasa Field Site was received from the University of Washington Center for AIDS Research (CFAR), an NIH funded program [P30 AI027757] which is supported by the following NIH Institutes and Centers (NIAID, NCI, NIMH, NIDA, NICHD, NHLBI, NCCAM).

We gratefully acknowledge support from our clinic staff, laboratory staff and administrators for their dedication and hard work. We express our appreciation to the Municipal Council of Mombasa and the administration of Coast Provincial General Hospital for use of their facilities. Finally, we sincerely thank the women whose time, effort, and commitment made this research possible.

\section{References}

1. Atashili J, Poole C, Ndumbe PM, Adimora AA, Smith JS. Bacterial vaginosis and HIV acquisition: a meta-analysis of published studies. AIDS. Jul 31; 2008 22(12):1493-1501. [PubMed: 18614873]

2. Larsson PG, Platz-Christensen JJ, Thejls H, Forsum U, Pahlson C. Incidence of pelvic inflammatory disease after first-trimester legal abortion in women with bacterial vaginosis after treatment with metronidazole: a double-blind, randomized study. Am J Obstet Gynecol. Jan; 1992 166(1 Pt 1):100103. [PubMed: 1733176]

3. Hillier SL, Nugent RP, Eschenbach DA, et al. Association between bacterial vaginosis and preterm delivery of a low-birth-weight infant. The Vaginal Infections and Prematurity Study Group. N Engl J Med. Dec 28; 1995 333(26):1737-1742. [PubMed: 7491137]

4. Gravett MG, Nelson HP, DeRouen T, Critchlow C, Eschenbach DA, Holmes KK. Independent associations of bacterial vaginosis and Chlamydia trachomatis infection with adverse pregnancy outcome. JAMA. Oct 10; 1986 256(14):1899-1903. [PubMed: 3761496]

5. Schwebke JR, Desmond R. A randomized trial of metronidazole in asymptomatic bacterial vaginosis to prevent the acquisition of sexually transmitted diseases. Am J Obstet Gynecol. Jun; 2007 196(6):517 e511-516. [PubMed: 17547876]

6. Centers for Disease Control and Prevention. Sexually Transmitted Diseases Treatment Guidelines. MMWR Recomm Rep. 2010; 59(RR-12):1-110.

7. Hillier, S. Bacterial Vaginosis. In: Holmes, KKSP.; Stamm, W.; Piot, P.; Wasserheit, J.; Corey, L.; Cohen, M.; Watts, D., editors. Sexually Transmitted Diseases. 4. New York: McGraw-Hill; 2008.

8. Bradshaw CS, Morton AN, Hocking J, et al. High Recurrence Rates of Bacterial Vaginosis over the Course of 12 Months after Oral Metronidazole Therapy and Factors Associated with Recurrence. The Journal of Infectious Diseases. 2006; 193(11):1478-1486. [PubMed: 16652274]

9. Sobel JD, Ferris D, Schwebke J, et al. Suppressive antibacterial therapy with $0.75 \%$ metronidazole vaginal gel to prevent recurrent bacterial vaginosis. Am J Obstet Gynecol. May; 2006 194(5):12831289. [PubMed: 16647911]

10. Wilson J. Managing recurrent bacterial vaginosis. Sex Transm Infect. Feb; 2004 80(1):8-11. [PubMed: 14755028]

11. Amsel R, Totten PA, Spiegel CA, Chen KC, Eschenbach D, Holmes KK. Nonspecific vaginitis. Diagnostic criteria and microbial and epidemiologic associations. Am J Med. Jan; 1983 74(1):1422. [PubMed: 6600371]

12. McClelland RS, Richardson BA, Hassan WM, et al. Improvement of vaginal health for Kenyan women at risk for acquisition of human immunodeficiency virus type 1: results of a randomized trial. J Infect Dis. May 15; 2008 197(10):1361-1368. [PubMed: 18444793] 
13. Martin HL Jr, Jackson DJ, Mandaliya K, et al. Preparation for AIDS vaccine evaluation in Mombasa, Kenya: establishment of seronegative cohorts of commercial sex workers and trucking company employees. AIDS Res Hum Retroviruses. 1994; 10( Suppl 2):S235-237. [PubMed: 7865309]

14. Nugent RP, Krohn MA, Hillier SL. Reliability of diagnosing bacterial vaginosis is improved by a standardized method of gram stain interpretation. J Clin Microbiol. Feb; 1991 29(2):297-301. [PubMed: 1706728]

15. Martin HL, Richardson BA, Nyange PM, et al. Vaginal Lactobacilli, Microbial Flora, and Risk of Human Immunodeficiency Virus Type 1 and Sexually Transmitted Disease Acquisition. The Journal of Infectious Diseases. 1999; 180(6):1863-1868. [PubMed: 10558942]

16. Eschenbach DA, Davick PR, Williams BL, et al. Prevalence of hydrogen peroxide-producing Lactobacillus species in normal women and women with bacterial vaginosis. J Clin Microbiol. Feb; 1989 27(2):251-256. [PubMed: 2915019]

17. Hassan WM, Lavreys L, Chohan V, et al. Associations between intravaginal practices and bacterial vaginosis in Kenyan female sex workers without symptoms of vaginal infections. Sex Transm Dis. Jun; 2007 34(6):384-388. [PubMed: 17065846]

18. Newton ER, Piper JM, Shain RN, Perdue ST, Peairs W. Predictors of the vaginal microflora. Am J Obstet Gynecol. Apr; 2001 184(5):845-853. discussion 853-845. [PubMed: 11303191]

19. Swidsinski A, Mendling W, Loening-Baucke V, et al. Adherent biofilms in bacterial vaginosis. Obstet Gynecol. Nov; 2005 106(5 Pt 1):1013-1023. [PubMed: 16260520]

20. Swidsinski A, Mendling W, Loening-Baucke V, et al. An adherent Gardnerella vaginalis biofilm persists on the vaginal epithelium after standard therapy with oral metronidazole. Am J Obstet Gynecol. Jan; 2008 198(1):97 e91-96. [PubMed: 18005928]

21. Marrazzo JM, Thomas KK, Fiedler TL, Ringwood K, Fredricks DN. Relationship of specific vaginal bacteria and bacterial vaginosis treatment failure in women who have sex with women. Ann Intern Med. Jul 1; 2008 149(1):20-28. [PubMed: 18591634]

22. Fredricks DN, Fiedler TL, Marrazzo JM. Molecular identification of bacteria associated with bacterial vaginosis. N Engl J Med. Nov 3; 2005 353(18):1899-1911. [PubMed: 16267321]

23. Ferris MJ, Masztal A, Aldridge KE, Fortenberry JD, Fidel PL Jr, Martin DH. Association of Atopobium vaginae, a recently described metronidazole resistant anaerobe, with bacterial vaginosis. BMC Infect Dis. Feb 13.2004 4:5. [PubMed: 15018635]

24. Gardner HL, Dukes CD. Haemophilus vaginalis vaginitis: a newly defined specific infection previously classified non-specific vaginitis. Am J Obstet Gynecol. May; 1955 69(5):962-976. [PubMed: 14361525]

25. Sanchez S, Garcia PJ, Thomas KK, Catlin M, Holmes KK. Intravaginal metronidazole gel versus metronidazole plus nystatin ovules for bacterial vaginosis: a randomized controlled trial. Am J Obstet Gynecol. Dec; 2004 191(6):1898-1906. [PubMed: 15592270]

26. Probiotics: the Potential for a Live Microbicide. 2010

27. Klebanoff SJ, Hillier SL, Eschenbach DA, Waltersdorph AM. Control of the microbial flora of the vagina by H2O2-generating lactobacilli. J Infect Dis. Jul; 1991 164(1):94-100. [PubMed: 1647428]

28. Senok AC, Verstraelen H, Temmerman M, Botta GA. Probiotics for the treatment of bacterial vaginosis. Cochrane Database Syst Rev. 2009; (4):CD006289. [PubMed: 19821358]

29. Marrazzo JM, Martin DH, Watts DH, et al. Bacterial vaginosis: identifying research gaps proceedings of a workshop sponsored by DHHS/NIH/NIAID. Sex Transm Dis. Dec; 2010 37(12): 732-744. [PubMed: 21068695] 


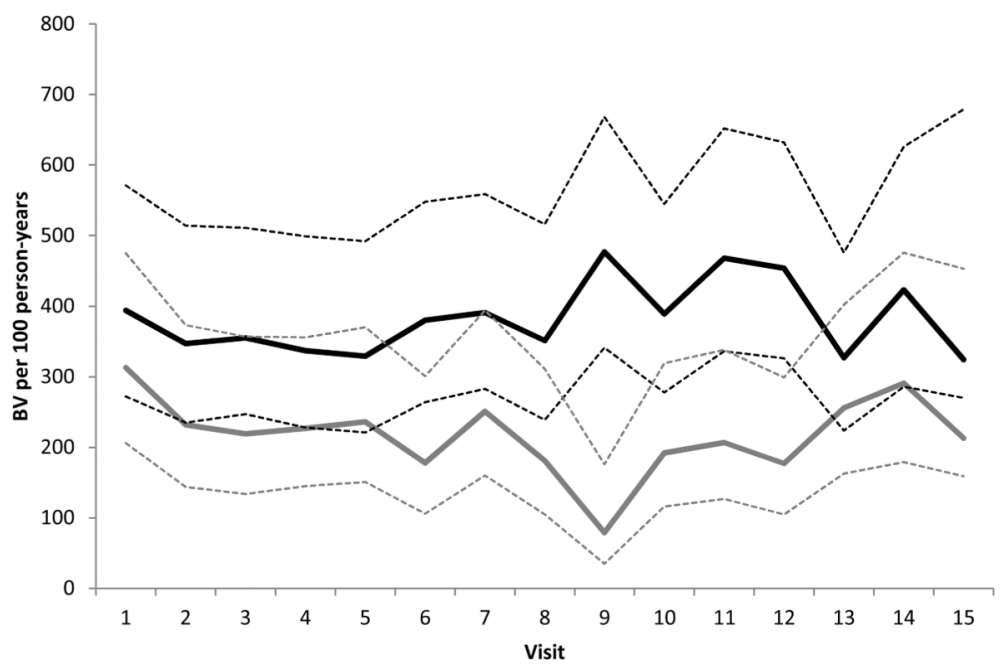

Figure 1.

Incidence of BV at each RCT visit and at the first three post-trial monthly study visits by RCT study arm ${ }^{1}$

${ }^{1}$ The incidence of BV at each study visit among the 165 women who completed all $12 \mathrm{RCT}$ visits and had at least one cohort study visit within 120 days of completing the RCT

_ Placebo _ _ _ $\_95 \%$ Confidence Interval

Intervention …........... 95\% Confidence Interval

The dashed vertical line represents the end of the RCT. Visits to the right of the dashed line occurred during the 120-day post-trial period. 
TABLE 1

Demographic and Clinical Characteristics of Participants at Baseline Visit for the Analysis (Corresponding to Final Randomized Controlled Trial Visit)

\begin{tabular}{|c|c|c|c|}
\hline & $\begin{array}{l}\text { Intervention } \\
\mathrm{n}=\mathbf{8 3}\end{array}$ & $\begin{array}{l}\text { Placebo } \\
n=82\end{array}$ & $P$ \\
\hline Age (yr) & $34(29-40)$ & $35(30-40)$ & 0.76 \\
\hline Education duration (yr) & $8(6-8)$ & $8(7-10)$ & 0.13 \\
\hline Duration of sex work (yr) & $7.6(4-13)$ & $6.2(3-11)$ & 0.17 \\
\hline Sex in the past week & $57(69)$ & $47(57)$ & 0.13 \\
\hline Unprotected sex in the past week ${ }^{*}$ & $16(19)$ & $23(28)$ & 0.19 \\
\hline Hormonal contraceptive use ${ }^{\dagger}$ & $23(28)$ & $24(29)$ & 0.83 \\
\hline Vaginal washing in the past week & $78(94)$ & $70(85)$ & 0.10 \\
\hline Bacterial vaginosis & $13(16)$ & $35(43)$ & $<0.001$ \\
\hline Abnormal vaginal flora ${ }^{*}$ & $27(33)$ & $50(61)$ & $<0.001$ \\
\hline Vaginal candidiasis & $5(6)$ & $4(5)$ & 0.75 \\
\hline Trichomonas vaginalis infection & $0(0)$ & $1(1)$ & 0.31 \\
\hline \multicolumn{4}{|l|}{ Lactobacillus colonization } \\
\hline Any species & $14(17)$ & $15(18)$ & 0.81 \\
\hline Hydrogen peroxide producers & $5(6)$ & $9(11)$ & 0.25 \\
\hline Neisseria gonorrhoeae infection & $0(0)$ & $1(1)$ & 0.31 \\
\hline Cervicitis $\xi$ & $2(2)$ & $2(2)$ & 0.98 \\
\hline
\end{tabular}

$\mathrm{n}(\%)$ or median (interquartile range) presented.

* Unprotected sex in the past week versus protected sex or no sex in the past week. Among 104 women reporting sex in the past week, $28 \%$ of women in the intervention arm reported unprotected sex compared with $49 \%$ in the placebo arm $(P=0.03)$.

${ }^{\dagger}$ DMPA or OCP use.

Nugent score $[\mathrm{mt}] 3$.

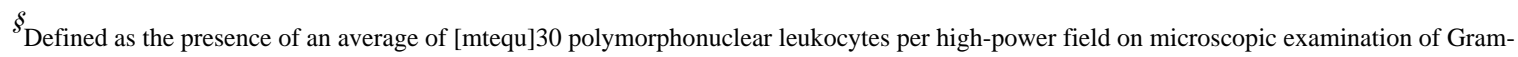
stained cervical secretions (original magnification [times]100). 
